\title{
Coronavirus Disease-19 Quarantine Experience in the Middle East Region: Emotional Status, Health Patterns, and Self-efficacy Survey
}

\author{
Rasha Mohammed Hussien ${ }^{1 *}$, Mahmoud Abdul Hameed Shahin ${ }^{2}$ \\ ${ }^{1}$ Department of Psychiatric and Mental Health Nursing, Faculty of Nursing, Zagazig University, Zagazig, Egypt; ${ }^{2}$ Assistant \\ professor of Critical Care Nursing, Department of Nursing, Al-Ghad International Colleges for Applied Medical Sciences, \\ Qassim, Saudi Arabia
}

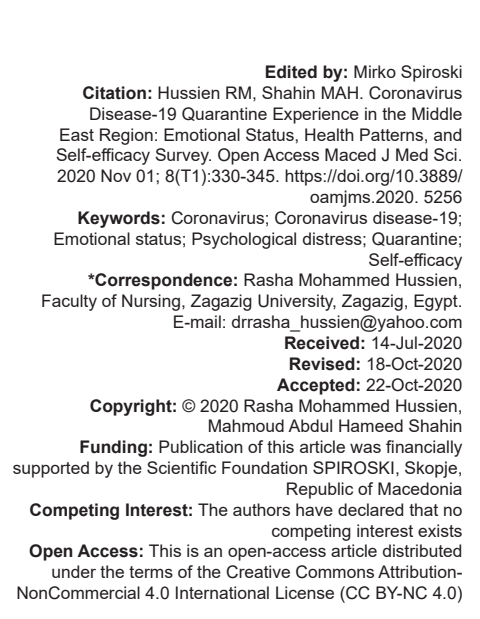

\section{Introduction}

Quarantine refers to the separation and restriction of movement of individuals who have been exposed to a contagious disease, to confirm whether they have been infected and reduce the chance that they will infect others [1]. Quarantine differs from isolation, which is the separation of individuals who have been diagnosed with contagious and infectious diseases from individuals who are not sick; however, the two phrases are frequently used interchangeably, particularly in the public discourse. In just a few months, the upward trajectory of coronavirus infections has closed schools, offices, stores, and factories. Airplanes have been grounded and borders have been closed

\begin{abstract}
(COVID)-19 pandemic can provoke anxiety, stress, sadness, and fear

AIM: The aim of the study was to assess the emotional states (depression, anxiety, and stress), functional health patterns, and self-efficacy among individuals from Egypt, Jordan, and Saudi Arabia during the home quarantine experience associated with COVID-19.

704 participants from Egypt, Jordan, and Saudi Arabia. Data collection was performed in April 2020, using a selfadministered questionnaire that consisted of a sociodemographic data sheet, the Depression, Anxiety, Stress Scale

for Egyptian participants compared with those from other countries. In contrast, participants from Egypt presented significantly reduced GSES scores than those from other countries. Higher mean DASS scores were identified among females, participants who reported inappropriate housing conditions and participants who were unemployed, young, widowed, and school-educated. A high mean DASS score was significantly associated with reported disturbances in sleep, sexuality, and social communication, work schedule changes, the inability to concentrate on positive thoughts, the inability to empty their brains of daily thoughts, and not caring about regular interactions with family. Increased mean GSES scores were significantly associated with males, participants who reported appropriate housing conditions, participants who live alone, are older, married, hold higher education degrees, and who are reduced appetites. However, a significant inverse correlation was detected between the mean DASS and GSES CONCLUSION: The COVID-19 quarantine was associated with a mild level of depression stress and normal anxiety levels, with higher psychological distress and lower self-efficacy identified among participants from Egypt. The emotional status of individuals during the COVID-19 pandemic should be explored further, and awareness programs, designed to address the psychological effects of quarantine, should be promoted, through mass media and othe means, with consideration of the effects on the general population, COVID-19 cases, individuals with close contacts with COVID-19 cases, and health-care professionals.
\end{abstract}

to travelers. Modern quarantine consists of a variety of disease prevention strategies that can be used individually or in combination, including a temporary, voluntary home curfew, the minimization and prevention of group meetings, the cancellation of public events, the closure of mass transit systems, and the implementation of other restrictions on international and cross-country travel [2].

Functional health patterns can be described as the ability to perform daily living activities. Gordon's functional health patterns supply a holistic model for the evaluation of families, with functional assessments classified under the following eleven headings: Health perception, nutrition, elimination, exercise, perceptual pattern, sleep, self-concept pattern, relationship pattern, sexuality, coping, and health belief pattern [3]. 
Various research has shown that human behavioral changes, such as reducing social contacts during outbreaks, can have considerable effects on disease spread [4]. This social distancing response can be especially beneficial during the early stages of an epidemic, when pharmaceutical interventions, such as antiviral medications and vaccinations, may not yet be conveniently available. Social distancing can also be enforced centrally, through the closure of faculties and offices and the cancellation of events, or emerge naturally, due to individual actions [5].

Recently, quarantine was instituted during the coronavirus disease 2019 (COVID-19) pandemic outbreak. During this outbreak, entire cities in China were effectively placed under mass quarantine restrictions and thousands of expatriated nationals who have returned home from China have been requested to self-isolate, either at home or in government-run facilities [6], [7], [8]. Many precedents exist for such measures, including the citywide quarantines that were imposed in areas of China and Canada during the 2003 outbreak of severe acute respiratory syndrome (SARS), and whole villages in many West African nations were quarantined during the 2014 Ebola outbreak, as reported by the Public Health England organization [6], [8].

The COVID-19 pandemic is both an epidemiological and psychological crisis. The current state of affairs can provoke anxiety, stress, sadness, and fear; therefore, the timely assessment of individual psychological health status is urgently necessary for society [9]. Previous studies have revealed profound and various psychosocial impacts on people, at the individual, community, and worldwide levels during infection outbreaks. On the individual level, people are likely to experience worry regarding sickness and death, experience internal feelings of helplessness, and stigma. During one influenza outbreak, approximately $10-30 \%$ of the general public were very or fairly concerned about the possibility of contracting the viral disease [10]. With the closure of faculties and business, the negative thoughts experienced by most individuals can be compounded [11].

Widespread outbreaks of infectious diseases, such as COVID-19, are generally associated with increased psychological stress. In addition to feelings of unpredictability and uncertainty, the seriousness of the disease, misinformation, and social isolation can contribute to stress and psychological sickness [12]. Another study revealed substantial concerns regarding the impacts of social isolation and social distancing on general well-being, and social isolation has been associated with increased anxiety, depression, stress, loneliness, self-harm, suicide attempts, and many other negative emotions. In addition, pandemic responses are also associated with financial difficulties, which can increase stress responses [13].

According to Bandura [14], self-efficacy plays a key role in the regulation of emotional states.
Self-efficacy beliefs facilitate the interpretation of potentially threatening expectations as manageable but considerable challenges, which allows individuals to experience reduced feelings of burden and stress during difficult situations. By decreasing the negative thoughts and worries associated with potential threats, individuals with self-efficacy can regulate and control their emotional states more effectively. In addition, various studies have identified a relationship between poor mental and psychiatric well-being and general self-efficacy, as individuals' beliefs in their own capabilities can affect their experiences of stress and despair [15], [16].

Many studies have validated the finding that self-efficacy is an independent predictor of mental health. Inverse relationships exist between self-efficacy and depression, stress, and emotional coping strategies [17], [18]. Other studies have shown that low self-efficacy expectations are correlated with the increased use of emotion-focused coping strategies, such as denial and self-criticism, which are associated with the signs and symptoms of despair, stress, psychosomatic disorders, and poor well-being. Moreover, self-efficacy has considerable consequences for essential aspects of personality and situationspecific behaviors that are associated with wellness (e.g., coping with stress and conflict resolution) and is regarded as an element that can protect an individual's health, lowering their risks of biological and psychological diseases [19], [20].

\section{Significance of the study}

Local and international health emergencies associated with the COVID-19 pandemic are likely to have negative impacts on the physical and psychological health of the population, increasing the incidence of psychological crises [9]. Crisis managers rely on public compliance during self-quarantine efforts. Officials often have limited abilities to implement and monitor these measures; hence, the public health benefits of household quarantine can only be achieved if the public cooperates.

Current research priorities include the monitoring and reporting of emotional status issues, such as depression, anxiety, and stress, both to recognize the mechanisms that drive these negative emotions and to identify necessary corrective interventions. Identifying functional health pattern changes that occur among the public during home quarantine is an important task that can reflect the general public health status and uncover susceptibility to some chronic diseases, such as diabetes, hypertension, and coronary heart disease. Functional health pattern assessments should be adopted throughout the general population, especially among vulnerable groups, including frontline healthcare workers. The assessment of emotional status, functional health pattern issues, and selfefficacy during the home quarantine experience 
associated with COVID-19 is, therefore, necessary. We will perform such an assessment on a large sample of the population, utilizing a comparative study design, to examine accessible countries in the Middle East region, including Egypt, Jordan, and Saudi Arabia. Saudi Arabia is a country in Western Asia that constitutes the bulk of the Arabian Peninsula, with a population of 34 million, as reported in 2019 [21]. Jordan is an Arab country in Western Asia, on the East Bank of the Jordan River, with a population of more than 10 million, as reported in 2020 [22]. However, Egypt is a transcontinental country that spans the northeast corner of Africa and the southwest corner of Asia, through a land bridge formed by the Sinai Peninsula, with over 100 million inhabitants, as reported in 2020 [23]. All three countries are located in the Middle East region.

\section{Methods}

\section{Design and setting}

A descriptive, cross-sectional, and comparative research design study was conducted, simultaneously, in Egypt, Jordan, and Saudi Arabia, during the COVID19 pandemic outbreak. These three countries were selected due to the convenience, accessibility, and availability of researchers in these countries.

\section{Study sample}

A convenience sample of the general populations of Egypt (198 participants), Jordan (148 participants), and Saudi Arabia (358 participants) were recruited. The sample size was calculated according to the proportionate populations of the three countries, at a $99 \%$ level of confidence, with $5 \%$ confidence limits, $50 \%$ anticipated frequency, and a design effect value of 1.0. Using the Open-Epi, V3 software package, the required sample size was determined to be 664 subjects, which was increased to 704 subjects to assure the achievement of the targeted confidence level.

\section{Data collection tools}

The researcher used a self-administered questionnaire form, which included scales to assess the emotional status and general self-efficacy, in addition to the personal characteristics of the study participants, including age, gender, marital status, work, monthly income, current living status, housing conditions, number of people living in the same dwelling, level of education, chronic illnesses, and the recurrence of infection. The questionnaire also included questions regarding changes in functional health patterns associated with home quarantine, such as smoking, exercise, appetite, body weight, sleep and sexuality, communication, job schedule, family meetings, and thinking and belief patterns, which were derived from the 11 functional health pattern categories [3].

The Depression Anxiety Stress Scales 21 (DASS-21) was utilized to assess the emotional status of individuals [24]. The whole questionnaire consisted of three reliable, 7-item scales, which utilized 4-point Likert scales to measure the extent to which each state has been experienced over the past week $(0=$ did not apply to me at all; 3 = applied to me very much or most of the time). Scores were calculated as described by a previous study, with questions $3,5,10,13,16,17$, and 21 forming the depression subscale, questions $2,4,7,9,15,19$, and 20 forming the anxiety subscale, and questions 1 , $6,8,11,12,14$, and 18 forming the stress subscale [25]. The mean score for each subscale was calculated (0-3), and the levels of depression, anxiety, and stress were determined based on the mean (normal: <0.6; mild: $0.6 \leq 1.2$; moderate: $1.2 \leq 1.8$; severe: $1.8 \leq 2.4$; and extremely severe: $2.4-3)$. The DASS-21 subscale reliability was tested repeatedly, using Cronbach's alpha, which revealed acceptable levels of subscale reliability (depression $=0.83$, anxiety $=0.78$, and stress $=0.87$ ) [26], [27]. The DASS-21 form and permission for use are available online [28]; however, we adopted the Arabic version of the DASS-21, with no modifications [29].

The General Self-Efficacy Scale (GSES), established by Schwarzer and Jerusalem [30], was adopted to assess the self-efficacy of participants with regards to COVID-19, in the current study. The GSES was adapted to investigate perceptions of personal competence, based on ten items that participants respond to using a four-point Likert format, ranging from " 1 = Not true about me" to " 4 = Totally true about me" [31]. The Cronbach's alpha value for GSES was 0.87 when including responses for all 10 items from the pilot study. The self-efficacy levels of participants were defined according to the total GSES score (low $=10-19$, moderate $=20-30$, and high $=31-40)$. The Arabic version of the GSES and permission for use is publicly available online, as provided by the original authors [32].

\section{Pilot study}

A pilot study was performed prior to the main study, to determine the clarity of the scales and the feasibility of the study. The pilot study was conducted on $10 \%$ of the calculated study sample to test the applicability of the data collection tool and the feasibility of the study. Based on the pilot study results, the average time necessary to respond to the tool, including all three scales, ranged from 15 to $20 \mathrm{~min}$, depending on the respondent's level of understanding and cooperation. Based on the pilot study results, the questionnaire was finalized. Since some modifications were made to the phrasing of some items included in the sociodemographic and functional health pattern assessments, the pilot subjects were not included in 
the main study sample. The pilot study was also used to assess the reliability of the scales used.

\section{Study procedure}

Similar to other countries around the world, the governments of Egypt, Jordan, and Saudi Arabia recommended the minimization of face-to-face interactions and asked the public to isolate themselves at home. Potential respondents for piloting and study were, therefore, electronically invited, through the researchers' social networks, friends, and colleagues. Data were collected using an electronic survey that took approximately 15-20 min to complete. The online questionnaire was designed using Google Forms and was sent to participants through various social media platforms (WhatsApp, Messenger, Facebook, and Imo application). The data handling procedures followed all required national data protection standards. The study did not include any deception, and participants were debriefed at the end of the survey. The study only collected non-personally identifiable data. Data collection occurred over 10 days (11 April-20 April 2020), after the World Health Organization (WHO) declared the COVID-19 outbreak to be a public health emergency of international concern. The duration of data collection was determined based on the time required to gather a sufficient number of responses to meet the predetermined sample size.

\section{Statistical analysis}

Data retrieved from Google Forms were collected, revised, and coded using a personal computer. Statistical analysis was performed using the Statistical Package for Social Sciences, version 26. Data are presented using descriptive statistics, as the mean and standard deviation or number and percentage. The mean and standard deviation were used for continuous variables, whereas number and percentages were used for categorical variables. The Shapiro-Wilk test indicated that the data were not normally distributed; therefore, the Kruskal-Wallis test was used to compare the mean DASS and GSES scores across all three countries. The Mann-Whitney $U$ test was used to identify significant differences in the mean DASS and GSES scores between two countries and to compare dichotomous sociodemographic and functional health pattern variables, such as gender, employment, smoking, sexual pattern changes, and others. The Mann-Whitney $U$ test and the KruskalWallis test are nonparametric tests that can be used to compare mean ranks values but cannot be used to compare medians or distributions. A Pearson's correlation coefficient test was used to examine correlations among the DASS subscales and between the DASS and GSES scores. Multiple linear regression analysis was used to determine whether the DASS subscales were independent predictors of GSES scores and to formulate a predictive linear equation.

\section{Ethical considerations}

The research proposal was submitted to the ethical committee at Al-Ghad International Colleges for Applied Medical Sciences in Saudi Arabia and was approved before commencing data collection. Participation in this study was completely voluntary, and participants did not receive any form of coercion or financial compensation. Participants were asked to read and approve the included informed consent form before answering the questionnaire. Before participating in this study, subjects were informed regarding the purpose and type of study, the researchers' contact information and affiliations, and their rights to refuse or withdraw at any time. Potential breaches of confidentiality were minimized by the use of survey identification numbers; however, no identifying information, including names, email addresses, or mobile numbers were requested from the participants, and their responses were completely anonymous. No harm or risk, except for discomfort or inconvenience, was expected as a result of completing the questionnaire, and participants were given the option of choosing "l'd prefer not to answer this question" when they decide to avoid responding to some embarrassing or inconvenient questions in the sociodemographic part of the survey. All ethical principles regarding medical research involving human subjects, in accordance with the Declaration of Helsinki, were followed [33]. In addition, official permission for the use of the DASS and GSES scales was granted by the original authors [24], [30].

\section{Results}

The results of the current study are summarized in twelve tables (Tables 1-12), which describe the main findings. Table 1, for example, describes the sociodemographic information for the sample, which revealed that slightly more than onethird of the study sample across all three countries (Jordan, Egypt, and Saudi Arabia) were aged between 30 and 39 years, with a mean age of $35.32 \pm 9.8$ years $(n=704)$. Approximately two-thirds of all participants were male, married, and employed $(60 \%, 70 \%$, and $73 \%$, respectively). In contrast, approximately half of the sample holds a bachelor's degree and has a medium family size, ranging from 4 to 6 members (50\% and $53 \%$, respectively). Most participants lived with their families and earn a sufficient monthly family income (86\% and $82 \%$, respectively) and reported living in appropriate housing conditions, with sufficient ventilation and lighting (94\%). Finally, $16.8 \%$ of the sample indicated that they have chronic diseases, with a higher percentage of participants from Saudi Arabia reporting the occurrence of hypertension, diabetes, and psychiatric illnesses $(7.8 \%, 5.6 \%$, and $3.4 \%$, respectively), compared to those in Jordan and Egypt, 
Table 1: Sociodemographic characteristics $(n=704)$

\begin{tabular}{|c|c|c|c|c|c|c|c|c|}
\hline \multirow[t]{3}{*}{ Sociodemographics } & \multicolumn{8}{|c|}{ Place of residence } \\
\hline & \multicolumn{2}{|c|}{ Jordan $(n=148)$} & \multicolumn{2}{|c|}{ Egypt $(n=198)$} & \multicolumn{2}{|c|}{ Saudi Arabia $(n=358)$} & \multicolumn{2}{|l|}{ Total $(n=704)$} \\
\hline & $\mathrm{n}$ & $\%$ & $\mathrm{n}$ & $\%$ & $\mathrm{n}$ & $\%$ & $\mathrm{n}$ & $\%$ \\
\hline \multicolumn{9}{|l|}{ Age categories } \\
\hline 24 years or younger & 6 & 4 & 36 & 18 & 58 & 16 & 100 & 14 \\
\hline $25-29$ years & 20 & 14 & 20 & 10 & 62 & 17 & 102 & 14 \\
\hline $30-34$ years & 32 & 22 & 38 & 19 & 60 & 17 & 130 & 18 \\
\hline $35-39$ years & 24 & 16 & 42 & 21 & 68 & 19 & 134 & 19 \\
\hline $40-44$ years & 22 & 14 & 32 & 16 & 54 & 15 & 108 & 15 \\
\hline $45-49$ years & 24 & 16 & 16 & 9 & 28 & 8 & 68 & 11 \\
\hline 50 and older & 20 & 14 & 14 & 7 & 28 & 8 & 62 & 9 \\
\hline Mean \pm SD (years) & $38.45 \pm 9.09$ & & $34.4 \pm 10.0$ & & $34.54 \pm 9.83$ & & $35.32 \pm 9.85$ & \\
\hline \multicolumn{9}{|l|}{ Gender } \\
\hline Female & 54 & 36 & 80 & 40 & 150 & 42 & 284 & 40 \\
\hline Male & 94 & 64 & 118 & 60 & 208 & 58 & 420 & 60 \\
\hline \multicolumn{9}{|l|}{ Marital status } \\
\hline Divorced & 4 & 3 & 8 & 4 & 10 & 3 & 22 & 3 \\
\hline Married & 112 & 76 & 148 & 75 & 236 & 66 & 496 & 70 \\
\hline Single & 30 & 20 & 38 & 19 & 110 & 30 & 178 & 26 \\
\hline Widow & 2 & 1 & 4 & 2 & 2 & 1 & 8 & 1 \\
\hline \multicolumn{9}{|l|}{ Current living status } \\
\hline Living alone & 14 & 9 & 16 & 8 & 68 & 19 & 98 & 14 \\
\hline Living within a family & 134 & 91 & 182 & 92 & 290 & 81 & 606 & 86 \\
\hline \multicolumn{9}{|c|}{ Housing conditions, in terms of sufficient ventilation and lighting } \\
\hline Appropriate & 142 & 96 & 186 & 94 & 332 & 93 & 660 & 94 \\
\hline Inappropriate & 6 & 4 & 12 & 6 & 26 & 7 & 44 & 6 \\
\hline \multicolumn{9}{|l|}{ Household size (number of people) } \\
\hline Small (3 or less) & 40 & 27 & 30 & 15 & 118 & 33 & 188 & 27 \\
\hline Medium (4-6) & 86 & 58 & 140 & 71 & 148 & 42 & 374 & 53 \\
\hline Large $(7-9)$ & 22 & 15 & 20 & 10 & 62 & 17 & 104 & 15 \\
\hline Too large (10 or more) & 0 & 0 & 8 & 4 & 30 & 8 & 38 & 5 \\
\hline \multicolumn{9}{|l|}{ Employment } \\
\hline Employed & 116 & 78 & 120 & 61 & 280 & 78 & 516 & 73 \\
\hline Unemployed & 32 & 22 & 78 & 39 & 78 & 22 & 188 & 27 \\
\hline \multicolumn{9}{|l|}{ Monthly family income } \\
\hline Sufficient & 118 & 80 & 142 & 72 & 320 & 89 & 580 & 82 \\
\hline Insufficient & 30 & 20 & 56 & 28 & 38 & 11 & 124 & 18 \\
\hline \multicolumn{9}{|l|}{ Educational level } \\
\hline Bachelor's degree & 54 & 36 & 92 & 46 & 208 & 58 & 354 & 50 \\
\hline Diploma & 32 & 22 & 60 & 30 & 16 & 4 & 108 & 15 \\
\hline Postgraduate studies & 42 & 28 & 28 & 14 & 106 & 30 & 176 & 25 \\
\hline School & 20 & 14 & 18 & 10 & 28 & 8 & 66 & 10 \\
\hline Has chronic diseases & 24 & 16.2 & 28 & 14.1 & 66 & 18.4 & 118 & 16.8 \\
\hline Has diabetes mellitus & 8 & 5.4 & 10 & 5.1 & 20 & 5.6 & 38 & 5.4 \\
\hline Has hypertension & 10 & 6.8 & 14 & 7.1 & 28 & 7.8 & 52 & 7.4 \\
\hline Has coronary artery disease or atherosclerosis & 6 & 4.10 & 8 & 4.00 & 12 & 3.30 & 26 & 3.70 \\
\hline Has psychiatric illness & 2 & 1.4 & 6 & 3.0 & 12 & 3.4 & 20 & 2.8 \\
\hline Has Recurrent infections & 12 & 8.1 & 6 & 3.0 & 18 & 5.0 & 36 & 5.1 \\
\hline
\end{tabular}

$\mathrm{N}=$ Sample size; $\mathrm{n}$ = frequency; \% = percent; $\mathrm{SD}=$ Standard deviation

Table 2: Functional health patterns $(n=704)$

\begin{tabular}{|c|c|c|c|c|c|c|c|c|}
\hline \multirow{3}{*}{ Functional health patterns } & \multicolumn{8}{|c|}{ Place of residence } \\
\hline & \multicolumn{2}{|c|}{ Jordan $(n=148)$} & \multicolumn{2}{|c|}{ Egypt $(n=198)$} & \multicolumn{2}{|c|}{ Saudi Arabia $(n=358)$} & \multicolumn{2}{|c|}{ Total $(n=704)$} \\
\hline & $\mathrm{n}$ & $\%$ & $\mathrm{n}$ & $\%$ & $\mathrm{n}$ & $\%$ & $\mathrm{n}$ & $\%$ \\
\hline \multicolumn{9}{|l|}{ Regular exercise } \\
\hline No & 112 & 75.7 & 164 & 82.8 & 232 & 64.8 & 508 & 72.2 \\
\hline Yes & 36 & 24.3 & 34 & 17.2 & 126 & 35.2 & 196 & 27.8 \\
\hline \multicolumn{9}{|l|}{ Smoke tobacco regularly } \\
\hline No & 92 & 62.2 & 162 & 81.8 & 292 & 81.6 & 546 & 77.6 \\
\hline Yes & 56 & 37.8 & 36 & 18.2 & 66 & 18.4 & 158 & 22.4 \\
\hline \multicolumn{9}{|l|}{ Appetite pattern } \\
\hline High & 36 & 24.3 & 34 & 17.2 & 64 & 17.9 & 134 & 19.0 \\
\hline Low & 6 & 4.1 & 24 & 12.1 & 34 & 9.5 & 64 & 9.1 \\
\hline Usual & 106 & 71.6 & 140 & 70.7 & 260 & 72.6 & 506 & 71.9 \\
\hline \multicolumn{9}{|l|}{ Body weight } \\
\hline Normal & 80 & 54.1 & 92 & 46.5 & 184 & 51.4 & 356 & 50.5 \\
\hline Overweight & 60 & 40.5 & 92 & 46.5 & 156 & 43.6 & 308 & 43.8 \\
\hline Underweight & 8 & 5.4 & 14 & 7 & 18 & 5.0 & 40 & 5.7 \\
\hline Suffer from sleeping disturbance due to quarantine? & 72 & 48.6 & 126 & 63.6 & 180 & 50.3 & 378 & 53.7 \\
\hline Suffer from sexuality disturbance due to quarantine? & 36 & 24.3 & 56 & 28.3 & 64 & 17.9 & 156 & 22.2 \\
\hline Suffer from social communication disturbances due to quarantine? & 80 & 54.1 & 140 & 70.7 & 184 & 51.4 & 404 & 57.4 \\
\hline Experienced changes in the nature or time schedule of your current job due to quarantine? & 102 & 68.9 & 174 & 87.9 & 294 & 82.1 & 570 & 81.0 \\
\hline Concentrate on positive thoughts during difficulties? & 116 & 78.4 & 108 & 54.5 & 282 & 78.8 & 506 & 71.9 \\
\hline Empty the brain of thoughts regarding tomorrow's schedule at bedtime? & 94 & 63.5 & 64 & 32.3 & 196 & 54.7 & 354 & 50.3 \\
\hline Care about meeting and communicating with your family on a daily basis? & 110 & 74.3 & 140 & 70.7 & 276 & 77.1 & 526 & 74.7 \\
\hline
\end{tabular}

whereas any infection recurrence was reported more frequently in Jordan (8.1\%) and Saudi Arabia (5\%) than in Egypt (3\%).

Table 2 shows the functional health patterns, demonstrating that the majority of the study sample does not engage in regular exercise, does not smoke, and has a normal appetite pattern $(72.2 \%, 77.6 \%$, and $71.9 \%$, respectively). Half of the participants have normal body weights $(50.6 \%)$, whereas slightly more than two-fifths $(43.8 \%)$ reported being overweight. Slightly more than half of the sample reported sleep and social communication disturbances associated with quarantine (53.7\% and $57.4 \%$, respectively). Approximately $49.7 \%$ of participants reported difficulty emptying their brains of thoughts at bedtime. In addition, $81 \%$ of the study sample reported recent changes in the nature or time 
Table 3: Difference in the mean depression, anxiety, stress, and DASS scores among the three countries $(\mathrm{n}=704)$

\begin{tabular}{|c|c|c|c|c|c|c|c|c|c|c|}
\hline DASS \& & Jordan $(n=148)$ & Egypt $(n=198)$ & Saudi Arabia $(n=358)$ & Total $(n=704)$ & Total level & Mean r & & & Kruskal-Wallis test & Sig. (2-tailed) \\
\hline Subscales & $\mathrm{M} \pm \mathrm{SD}$ & $\mathrm{M} \pm \mathrm{SD}$ & $\mathrm{M} \pm \mathrm{SD}$ & $\mathrm{M} \pm \mathrm{SD}$ & & Jordan & Egypt & KSA & Chi-Square & \\
\hline Depression & $0.57 \pm 0.47$ & $0.84 \pm 0.65$ & $0.63 \pm 0.60$ & $0.68 \pm 0.60$ & Mild & 325.45 & 405.21 & 334.53 & 18.9 & $0.000^{* *}$ \\
\hline Anxiety & $0.32 \pm 0.40$ & $0.56 \pm 0.58$ & $0.42 \pm 0.55$ & $0.43 \pm 0.54$ & Normal & 319.23 & 399.31 & 340.37 & 16.410 & $0.000^{* *}$ \\
\hline Stress & $0.67 \pm 0.58$ & $1.06 \pm 0.77$ & $0.74 \pm 0.67$ & $0.82 \pm 0.70$ & Mild & 317.49 & 420.12 & 329.58 & 31.036 & $0.000^{* *}$ \\
\hline DASS & $0.52 \pm 0.43$ & $0.82 \pm 0.61$ & $0.60 \pm 0.55$ & $0.64 \pm 0.56$ & Mild & 318.55 & 413.53 & 332.78 & 25.365 & $0.000^{* *}$ \\
\hline
\end{tabular}

Table 4: Differences in the mean GSES scores across countries $(n=704)$

\begin{tabular}{|c|c|c|c|c|c|c|c|c|c|c|}
\hline \multirow[t]{2}{*}{ GSES } & Jordan $(n=148)$ & Egypt $(n=198)$ & $\mathrm{KSA}(n=358)$ & Total $(\mathrm{N}=704)$ & Total level & \multicolumn{3}{|c|}{ Mean rank } & Kruskal-Wallis test & \multirow[t]{2}{*}{ Sig. (2-tailed) } \\
\hline & $\mathrm{M} \pm \mathrm{SD}$ & $\mathrm{M} \pm \mathrm{SD}$ & $\bar{M} \pm \mathrm{SD}$ & $\mathrm{M} \pm \mathrm{SD}$ & & Jordan & Egypt & KSA & Chi-square & \\
\hline Total of general self-efficacy scale & $32.16 \pm 5.18$ & $29.22 \pm 6.03$ & $32.38 \pm 5.57$ & $31.45 \pm 5.79$ & High & 376.65 & 274.69 & 385.55 & 40.688 & $0.000^{* *}$ \\
\hline
\end{tabular}

schedule of their current jobs due to quarantine. Most of the participants reported that they do care about meeting and communicating with their families on a daily basis and that they concentrate on positive thoughts during difficulties (74.7\% and $71.9 \%$, respectively). Almost onefifth of participants reported sexual pattern disturbances, due to home quarantine $(22.2 \%)$.

Table 3 revealed mild levels of depression and stress and low total DASS scores, for the entire sample, with normal anxiety levels, and significant differences were found when comparing the DASS scores among the three countries $(p<0.01)$. Participants from Egypt demonstrated significantly higher mean scores for depression, anxiety, stress, and total DASS compared with those from Saudi Arabia and Jordan.

A significant difference in the mean GSES scores was found among the participants of the three countries, as shown in Table 4. At the time of the survey, participants from Egypt displayed a moderate level of self-efficacy during home quarantine, whereas participants from Jordan and Saudi Arabia showed high self-efficacy levels $(p<0.01)$.

The Mann-Whitney $U$ test was used to identify significant differences between the mean scores of the DASS total score, the DASS depression, anxiety, and stress subscale scores, and the GSES scores when two countries were compared directly. Table 5 shows that highly significant differences were observed for the mean depression, anxiety, and stress subscale scores, the mean total DASS scores, and the mean GSES scores between Egyptian and Jordanian participants and between Saudi Arabian and Egyptian participants $(p<0.01)$. Nonetheless, no significant difference was observed between the Jordanian and Saudi participants in that regard.

Table 5: Matrix of differences between the mean scale and subscale scores between countries

\begin{tabular}{llll}
\hline Mann-Whitney U test Asymp. Sig. (2-tailed) & Scale & Egypt & Saudi Arabia \\
\hline Jordan & Depression & $0.000^{\star *}$ & 0.708 \\
& Anxiety & $0.000^{\star *}$ & 0.292 \\
& Stress & $0.000^{\star *}$ & 0.581 \\
& DASS & $0.000^{\star *}$ & 0.564 \\
& GSES & $0.000^{\star *}$ & 0.557 \\
\multirow{3}{*}{ Egypt } & Depression & - & $0.000^{* *}$ \\
& Anxiety & - & $0.001^{* *}$ \\
& Stress & - & $0.000^{* *}$ \\
& DASS & - & $0.000^{\star *}$ \\
& GSES & - & $0.000^{* *}$ \\
\hline${ }^{*}$ Significant at the 0.01 level (2-tailed). DASS: Depression, Anxiety, Stress Scale. GSES: General Self-
\end{tabular}

Efficacy Scale.
A high DASS mean score was significantly associated with the female gender, participants who reported inappropriate housing conditions, and unemployed participants ( $p<0.05$ ), as shown in Table 6. Significant differences were found in DASS mean scores according to age, marital status, the number of household members, and the educational level of participants. Using the Mann-Whitney $U$ test, DASS mean scores were found to be significantly higher for participants younger than 24 years compared with those who were older than 45 years. Widowed participants had higher DASS mean scores compared with both married and single participants. Participants from medium family sizes (4-6 members) had higher DASS mean scores compared with those from large and too large family sizes (7 members or more). Finally, participants who had a basic school education had higher DASS mean scores than participants with all other levels of education (Diploma, Bachelor's degree, and Postgraduate).

Similarly, a high GSES mean score was significantly associated with the male gender, participants who reported appropriate housing conditions, participants who lived alone, employed participants, and participants with sufficient monthly incomes $(p<0.05)$, as shown in Table 7. Significant differences were found in the GSES mean scores according to age category, marital status, and educational level. Using the MannWhitney $U$ test, GSES mean scores were found to be significantly higher for older participants (50 years and older) compared with participants younger than 24 years. GSES scores were also higher for married participants compared with single and divorced participants. GSES scores increased with education, as participants with postgraduate educations presented higher GSES scores than those with bachelor's degrees, diplomas, and basic school educations, bachelor's degree holders presented high scores than participants with diplomas and basic school educations, and diploma holders presented higher scores than basic school-educated participants.

A high DASS mean score was significantly associated with reporting disturbances in sleep, sexuality, social communication, and job schedules, due to quarantine, among participants (Table 8). Higher DASS mean scores were reported for participants who reported 
Table 6: Differences in DASS mean scores according to sociodemographic categories

\begin{tabular}{|c|c|c|c|c|}
\hline Sociodemographics & $\mathrm{M} \pm \mathrm{SD}$ & Mean rank & Test & $\mathrm{p}$-value \\
\hline \multicolumn{5}{|l|}{ Age categories } \\
\hline 24 years or less & $0.84 \pm 0.69$ & 408.80 & \multirow[t]{7}{*}{ Kruskal-Wallis test Chi-square $=24.17$} & \multirow[t]{7}{*}{$0.000^{\star *}$} \\
\hline $25-29$ years & $0.64 \pm 0.51$ & 357.74 & & \\
\hline $30-34$ years & $0.66 \pm 0.47$ & 379.02 & & \\
\hline $35-39$ years & $0.62 \pm 0.53$ & 341.63 & & \\
\hline $40-44$ years & $0.63 \pm 0.58$ & 348.30 & & \\
\hline $45-49$ years & $0.44 \pm 0.48$ & 265.15 & & \\
\hline 50 and more & $0.56 \pm 0.52$ & 324.08 & & \\
\hline \multicolumn{5}{|l|}{ Gender } \\
\hline Female & $0.71 \pm 0.60$ & 371.08 & Mann-Whitney U = 54364 & $0.046^{*}$ \\
\hline \multicolumn{5}{|l|}{ Marital status } \\
\hline Single & $0.70 \pm 0.58$ & 376.71 & \multirow[t]{4}{*}{ Kruskal-Wallis test Chi-square $=9.44$} & \multirow[t]{4}{*}{$0.024^{*}$} \\
\hline Married & $0.60 \pm 0.54$ & 339.52 & & \\
\hline Divorced & $0.71 \pm 0.47$ & 398.77 & & \\
\hline Widow & $1.08 \pm 0.68$ & 491.50 & & \\
\hline \multicolumn{5}{|l|}{ Current living status } \\
\hline Living alone & $0.68 \pm 0.57$ & 367.09 & \multirow[t]{2}{*}{ Mann-Whitney U = 28264} & \multirow[t]{2}{*}{0.44} \\
\hline Living within a family & $0.63 \pm 0.55$ & 350.14 & & \\
\hline \multicolumn{5}{|c|}{ Housing conditions, in terms of sufficient ventilation and lighting } \\
\hline Appropriate & $0.63 \pm 0.55$ & 348.34 & Mann-Whitney U = 11776 & $0.035^{*}$ \\
\hline Small (3 or less) & $0.66 \pm 0.54$ & 364.05 & \multirow[t]{4}{*}{ Kruskal-Wallis test Chi-square $=12.52$} & \multirow[t]{4}{*}{$0.006^{\star \star}$} \\
\hline Medium (4-6) & $0.68 \pm 0.58$ & 366.20 & & \\
\hline Large $(7-9)$ & $0.52 \pm 0.48$ & 312.12 & & \\
\hline Too large ( 10 or more) & $0.43 \pm 0.45$ & 271.08 & & \\
\hline \multicolumn{5}{|l|}{ Employment } \\
\hline Unemployed & $0.82 \pm 0.61$ & 418.37 & \multirow[t]{2}{*}{ Mann-Whitney U = 36120} & \multirow[t]{2}{*}{$0.000^{* *}$} \\
\hline Employed & $0.57 \pm 0.52$ & 328.50 & & \\
\hline \multicolumn{5}{|l|}{ Monthly family income } \\
\hline Insufficient & $0.68 \pm 0.56$ & 369.81 & \multirow[t]{2}{*}{ Mann-Whitney U = 33814} & \multirow[t]{2}{*}{0.296} \\
\hline Sufficient & $0.63 \pm 0.55$ & 348.80 & & \\
\hline \multicolumn{5}{|l|}{ Educational level } \\
\hline School & $0.81 \pm 0.59$ & 423.53 & \multirow[t]{4}{*}{ Kruskal-Wallis test Chi-square $=9.43$} & $0.024^{*}$ \\
\hline Diploma & $0.62 \pm 0.58$ & 344.64 & & \\
\hline Bachelor's degree & $0.63 \pm 0.55$ & 349.77 & & \\
\hline Postgraduate studies & $0.59 \pm 0.48$ & 336.17 & & \\
\hline Has chronic diseases & & & & \\
\hline No & $0.62 \pm 0.53$ & 346.44 & Mann-Whitney U = 31024 & 0.078 \\
\hline Yes & $0.75 \pm 0.64$ & 382.58 & & \\
\hline
\end{tabular}

Table 7: Difference in GSES mean scores according to the sociodemographic characteristics

\begin{tabular}{|c|c|c|c|c|}
\hline Sociodemographics & $\mathrm{M} \pm \mathrm{SD}$ & Mean rank & Test & p-value \\
\hline \multicolumn{5}{|l|}{ Age categories } \\
\hline 24 years or less & $29.80 \pm 5.4$ & 282.00 & \multirow[t]{7}{*}{ Kruskal-Wallis test Chi-square $=33.72$} & \multirow[t]{7}{*}{$0.000^{* *}$} \\
\hline $25-29$ years & $30.73 \pm 5.43$ & 329.48 & & \\
\hline $30-34$ years & $30.56 \pm 6.5$ & 321.07 & & \\
\hline $35-39$ years & $32.41 \pm 5.96$ & 388.31 & & \\
\hline $40-44$ years & $31.85 \pm 5.53$ & 370.11 & & \\
\hline $45-49$ years & $31.94 \pm 5.46$ & 372.21 & & \\
\hline 50 and more & $33.71 \pm 5.33$ & 440.31 & & \\
\hline \multicolumn{5}{|l|}{ Gender } \\
\hline Female & $30.71 \pm 6.23$ & 332.38 & Mann-Whitney U = 53926 & $0.031^{*}$ \\
\hline \multicolumn{5}{|l|}{ Marital status } \\
\hline Single & $30.38 \pm 6.32$ & 315.92 & \multirow[t]{4}{*}{ Kruskal-Wallis test Chi-square $=10.75$} & \multirow[t]{4}{*}{$0.013^{*}$} \\
\hline Married & $31.92 \pm 5.43$ & 368.38 & & \\
\hline Divorced & $29.54 \pm 7.18$ & 292.41 & & \\
\hline Widow & $30.75 \pm 7.3$ & 347.50 & & \\
\hline \multicolumn{5}{|l|}{ Current living status } \\
\hline Living alone & $32.65 \pm 5.42$ & 390.62 & \multirow[t]{2}{*}{ Mann-Whitney U = 25958} & \multirow[t]{2}{*}{$0.045^{*}$} \\
\hline Living within a family & $31.25 \pm 5.82$ & 346.33 & & \\
\hline \multicolumn{5}{|c|}{ Housing condition, in term of ventilation and lighting } \\
\hline Appropriate & $31.68 \pm 5.61$ & 359.30 & Mann-Whitney U = 10032 & $0.000^{* \star}$ \\
\hline Small (3 or less) & $32.17 \pm 5.45$ & 373.72 & \multirow[t]{4}{*}{ Kruskal-Wallis test Chi-square $=6.38$} & \multirow[t]{4}{*}{0.095} \\
\hline Medium (4-6) & $30.89 \pm 5.98$ & 334.91 & & \\
\hline Large (7-9) & $31.67 \pm 5.83$ & 364.50 & & \\
\hline Too large (10 or more) & $32.63 \pm 4.76$ & 387.82 & & \\
\hline \multicolumn{5}{|l|}{ Employment } \\
\hline Unemployed & $29.60 \pm 5.99$ & 288.80 & \multirow[t]{2}{*}{ Mann-Whitney U = 36528} & \multirow[t]{2}{*}{$0.000^{* *}$} \\
\hline Employed & $32.11 \pm 5.56$ & 375.71 & & \\
\hline \multicolumn{5}{|l|}{ Monthly family income } \\
\hline Insufficient & $30.38 \pm 5.90$ & 317.50 & \multirow[t]{2}{*}{ Mann-Whitney U = 31620} & \multirow[t]{2}{*}{$0.034^{*}$} \\
\hline Sufficient & $31.67 \pm 5.74$ & 359.98 & & \\
\hline \multicolumn{5}{|l|}{ Educational level } \\
\hline School & $28.3 \pm 6.02$ & 247.02 & \multirow[t]{4}{*}{ Kruskal-Wallis test Chi-square $=39.96$} & $0.000^{\star *}$ \\
\hline Diploma & $29.94 \pm 5.86$ & 302.09 & & \\
\hline Bachelor & $31.66 \pm 5.53$ & 357.87 & & \\
\hline Postgraduate studies & $33.11 \pm 5.49$ & 412.19 & & \\
\hline Has chronic diseases & & & & \\
\hline No & $31.41 \pm 5.76$ & 350.78 & Mann-Whitney U = 33564 & 0.616 \\
\hline Yes & $31.61 \pm 5.92$ & 361.06 & & \\
\hline
\end{tabular}


Table 8: DASS mean score difference according to participants' health patterns

\begin{tabular}{|c|c|c|c|c|}
\hline Health pattern & $\mathrm{M} \pm \mathrm{SD}$ & Mean rank & Test & $\mathrm{p}$-value \\
\hline \multicolumn{5}{|c|}{ Regular exercise } \\
\hline No & $0.65 \pm 0.55$ & 182282.00 & \multirow[t]{2}{*}{ Mann-Whitney U = 46572} & \multirow[t]{2}{*}{0.184} \\
\hline Yes & $0.60 \pm 0.57$ & 65878.00 & & \\
\hline \multicolumn{5}{|c|}{ Smoke tobacco regularly } \\
\hline No & $0.72 \pm 0.58$ & 187893.00 & \multirow[t]{2}{*}{ Mann-Whitney U = 38562} & \multirow[t]{2}{*}{0.192} \\
\hline Yes & $0.61 \pm 0.54$ & 60267.00 & & \\
\hline \multicolumn{5}{|l|}{ Appetite } \\
\hline Low & $1.02 \pm 0.60$ & 483.63 & \multirow{3}{*}{ Kruskal-Wallis test Chi-square $=29.47$} & \multirow[t]{3}{*}{$0.000^{* *}$} \\
\hline Usual & $0.61 \pm 0.54$ & 341.03 & & \\
\hline High & $0.58 \pm 0.52$ & 333.19 & & \\
\hline Underweight & $0.78 \pm 0.47$ & 432.95 & \multirow[t]{3}{*}{ Kruskal-Wallis test Chi-square $=7.150$} & \multirow[t]{3}{*}{$0.028^{*}$} \\
\hline Normal & $0.61 \pm 0.54$ & 342.46 & & \\
\hline Overweight & $0.65 \pm 0.57$ & 353.66 & & \\
\hline \multicolumn{5}{|c|}{ Disturbance in sleep due to quarantine? } \\
\hline No & $0.47 \pm 0.48$ & 283.41 & \multirow[t]{2}{*}{ Mann-Whitney U = 39090} & \multirow[t]{2}{*}{0.000 ** } \\
\hline Yes & $0.78 \pm 0.57$ & 412.09 & & \\
\hline \multicolumn{5}{|c|}{ Disturbance in sexuality due to quarantine? } \\
\hline No & $0.58 \pm 0.54$ & 327.62 & \multirow[t]{2}{*}{ Mann-Whitney U = 29110} & \multirow{2}{*}{$0.000^{* *}$} \\
\hline Yes & $0.85 \pm 0.56$ & 439.90 & & \\
\hline \multicolumn{5}{|c|}{ Disturbance in social communications due to quarantine? } \\
\hline \multicolumn{5}{|c|}{ Experienced changes in the nature or time schedule of your current job due to quarantine? } \\
\hline No & $0.48 \pm 0.49$ & 288.38 & \multirow[t]{2}{*}{ Mann-Whitney U = 29598} & \multirow[t]{2}{*}{$0.000^{\star *}$} \\
\hline Yes & $0.68 \pm 0.56$ & 367.57 & & \\
\hline \multicolumn{5}{|c|}{ Concentrate on positive thoughts during difficulties? } \\
\hline No & $1.04 \pm 0.61$ & 491.35 & \multirow[t]{2}{*}{ Mann-Whitney U = 22602} & \multirow[t]{2}{*}{$0.000^{* *}$} \\
\hline Yes & $0.48 \pm 0.44$ & 298.17 & & \\
\hline \multicolumn{5}{|c|}{ Empty your brain of thoughts regarding tomorrow's schedule at bedtime? } \\
\hline I cannot & $0.85 \pm 0.58$ & 434.48 & \multirow[t]{2}{*}{ Mann-Whitney U = 33258} & $0.000^{* *}$ \\
\hline I can & $0.43 \pm 0.43$ & 271.45 & & \\
\hline Care about mee & & & & \\
\hline No & $0.87 \pm 0.59$ & 437.92 & Mann-Whitney U = 31610 & $0.000^{\star *}$ \\
\hline Yes & $0.56 \pm 0.52$ & 323.60 & & \\
\hline
\end{tabular}

Table 9: Difference in GSES mean-scores based on participants health patterns

\begin{tabular}{|c|c|c|c|c|}
\hline Functional health patterns & $\mathrm{M} \pm \mathrm{SD}$ & Mean rank & Test & p-value \\
\hline \multicolumn{5}{|l|}{ Regular exercise } \\
\hline No & $32.42 \pm 5.65$ & 172124.00 & \multirow[t]{2}{*}{ Mann-Whitney U = 42838} & \multirow[t]{2}{*}{$0.004^{\star *}$} \\
\hline Yes & $31.06 \pm 5.80$ & 76036.00 & & \\
\hline \multicolumn{5}{|l|}{ Smoke tobacco regularly } \\
\hline No & $31.60 \pm 5.75$ & 195621.00 & \multirow[t]{2}{*}{ Mann-Whitney U = 39978} & \multirow[t]{2}{*}{0.16} \\
\hline Yes & $30.91 \pm 5.90$ & 52539.00 & & \\
\hline \multicolumn{5}{|l|}{ Appetite } \\
\hline Low & $28.87 \pm 6.89$ & 280.81 & \multirow[t]{3}{*}{ Kruskal-Wallis test Chi-square $=9.313$} & \multirow[t]{3}{*}{$0.011^{*}$} \\
\hline Usual & $31.83 \pm 5.38$ & 362.67 & & \\
\hline High & $31.22 \pm 6.39$ & 348.32 & & \\
\hline \multicolumn{5}{|l|}{ Body weight } \\
\hline Underweight & $29.70 \pm 7.43$ & 317.90 & \multirow[t]{3}{*}{ Kruskal-Wallis test Chi-square $=1.262$} & \multirow[t]{3}{*}{0.532} \\
\hline Normal & $31.52 \pm 5.57$ & 353.33 & & \\
\hline Overweight & $31.58 \pm 5.77$ & 356.04 & & \\
\hline \multicolumn{5}{|c|}{ Disturbance in sleep due to quarantine? } \\
\hline No & $31.76 \pm 5.86$ & 363.60 & \multirow[t]{2}{*}{ Mann-Whitney U=57996 } & \multirow[t]{2}{*}{0.178} \\
\hline Yes & $31.17 \pm 5.71$ & 342.93 & & \\
\hline \multicolumn{5}{|c|}{ Disturbance in sexuality due to quarantine? } \\
\hline No & $31.65 \pm 5.83$ & 361.08 & \multirow[t]{2}{*}{ Mann-Whitney U = 38040} & \multirow[t]{2}{*}{$0.035^{*}$} \\
\hline Yes & $30.71 \pm 5.57$ & 322.35 & & \\
\hline \multicolumn{5}{|c|}{ Disturbance in social communication due to quarantine? } \\
\hline No & $33.06 \pm 5.14$ & 408.45 & \multirow[t]{2}{*}{ Mann-Whitney U = 43814} & \multirow[t]{2}{*}{$0.000^{* *}$} \\
\hline Yes & $30.24 \pm 5.94$ & 310.95 & & \\
\hline \multicolumn{5}{|c|}{ Experienced changes in the nature or time schedule of your current job due to quarantine? } \\
\hline No & $31.86 \pm 5.75$ & 365.32 & \multirow[t]{2}{*}{ Mann-Whitney U = 36472} & \multirow[t]{2}{*}{0.416} \\
\hline Yes & $31.34 \pm 5.79$ & 349.49 & & \\
\hline \multicolumn{5}{|c|}{ Concentrate on positive thoughts during difficulties? } \\
\hline No & $28.46 \pm 5.48$ & 243.77 & \multirow[t]{2}{*}{ Mann-Whitney U = 28566} & \multirow[t]{2}{*}{$0.000^{* *}$} \\
\hline Yes & $32.61 \pm 5.48$ & 395.05 & & \\
\hline \multicolumn{5}{|c|}{ Empty your brain of thoughts regarding tomorrow's schedule at bedtime? } \\
\hline I cannot & $29.96 \pm 6.13$ & 301.05 & \multirow[t]{2}{*}{ Mann-Whitney U = 43942} & $0.000^{* *}$ \\
\hline I can & $32.90 \pm 5.02$ & 403.37 & & \\
\hline Care about meeting and $\mathrm{co}$ & & & & \\
\hline No & $28.56 \pm 5.93$ & 253.54 & Mann-Whitney U = 29200 & $0.000^{* *}$ \\
\hline Yes & $32.42 \pm 5.40$ & 385.99 & & \\
\hline
\end{tabular}

the inability to concentrate on positive thoughts during difficulties, participants who reported the inability to empty their brains of thoughts at bedtime, and participants who reported no interest in meeting or communicating with their families on a daily basis $(p<0.05)$. The MannWhitney $U$ test revealed that participants who suffered from low appetite had significantly higher DASS mean scores compared with those for participants with usual or increased appetites. Underweight participants had significantly increased DASS mean scores compared with both normal-weight and overweight participants.

A lower GSES mean score was significantly associated with participants who reported engaging in regular exercise and experiencing disturbances in sexuality and social communication due to quarantine (Table 9). However, a higher GSES mean score was 
found among participants who reported concentrating on positive thoughts during difficulties, who were able to empty their brains of thoughts at bedtime, and who cared about meeting and communicating with their families on a daily basis $(p<0.01)$. Using the MannWhitney $U$ test, participants who had low appetite were found to have significantly lower GSES mean scores compared with participants with usual and increased appetites.

Table 10: Correlations among the depression, anxiety, and stress subscale scores

\begin{tabular}{llll}
\hline & Mean depression & Mean anxiety & Mean stress \\
\hline $\begin{array}{l}\text { Mean depression } \\
\text { Pearson correlation }\end{array}$ & 1 & $0.721^{* *}$ & $0.773^{* *}$ \\
$\quad$ Sig. (2-tailed) & & 0.000 & 0.000 \\
n & 704 & 704 & 704 \\
Mean anxiety & & 1 & $0.734^{* *}$ \\
$\quad$ Pearson correlation & $0.721^{* *}$ & & 0.000 \\
Sig. (2-tailed) & 0.000 & 704 & 704 \\
n & 704 & & \\
Mean stress & & $0.734^{* *}$ & 1 \\
$\quad$ Pearson correlation & $0.773^{* *}$ & 0.000 & 704 \\
$\quad$ Sig. (2-tailed) & 0.000 & 704 & \\
n & 704 & & \\
${ }^{* *}$ Correlation is significant at the 0.01 level (2-tailed). &
\end{tabular}

Pearson's correlation coefficient indicated significant correlations among the mean depression, anxiety, and stress subscale scores $(p<0.01)$. As shown in Table 10, a strong positive correlation was identified among the three variables, in which an increase in the mean score of any one subscale was significantly associated with an increase in the mean scores of the two other subscales.

Table 11: Correlation between DASS and GSES scores

\begin{tabular}{|c|c|c|}
\hline & Mean DASS & Mean GSES \\
\hline \multicolumn{3}{|l|}{ Mean DASS } \\
\hline Pearson correlation & 1 & $-0.526^{* \prime}$ \\
\hline Sig. (2-tailed) & & 0.000 \\
\hline $\mathrm{n}$ & 704 & 704 \\
\hline \multicolumn{3}{|l|}{ Mean GSES } \\
\hline Pearson correlation & $-0.526^{\star \star}$ & 1 \\
\hline Sig. (2-tailed) & 0.000 & \\
\hline $\mathrm{n}$ & 704 & 704 \\
\hline
\end{tabular}

Table 11 illustrates a significant inverse correlation between the mean DASS and GSES scores $(p<0.01)$, indicating that increases in the mean DASS scores were associated with decreases in the mean GSES scores.

Table 12: Regression analysis of DASS subscale and GSES scores

\begin{tabular}{|c|c|c|c|c|c|}
\hline \multirow[t]{2}{*}{ Model } & \multicolumn{2}{|c|}{$\begin{array}{l}\text { Unstandardized } \\
\text { coefficients }\end{array}$} & \multirow{2}{*}{$\begin{array}{l}\text { Standardized } \\
\text { coefficients } \\
\text { Beta }\end{array}$} & \multirow[t]{2}{*}{$\mathrm{t}$} & \multirow[t]{2}{*}{ Sig. } \\
\hline & $\bar{B}$ & Std. error & & & \\
\hline (Constant) & 35.028 & 0.296 & & 118.321 & $0.000^{\star *}$ \\
\hline Mean depression scale & -1.675 & 0.522 & -0.174 & -3.210 & $0.001^{* *}$ \\
\hline Mean anxiety scale & -1.396 & 0.544 & -0.130 & -2.565 & $0.011^{*}$ \\
\hline Mean stress scale & -2.249 & 0.457 & -0.272 & -4.916 & $0.000^{* *}$ \\
\hline
\end{tabular}

Table 12 shows that the depression, anxiety, and stress subscale scores were significant independent predictors of the mean GSES score $(p<0.01)$. Based on the significance of the regression coefficient, the following linear regression equation was formulated, to predict the GSES mean-score from the depression, anxiety, and stress subscale mean scores, as follows: $y=35.028-1.675 x^{1}-1.396 x^{2}-2.249 x^{3}$, where 35.028 is a constant, $y$ is the mean GSES score, $x^{1}$ is the mean depression scale score, $x^{2}$ is the mean anxiety scale score, and $x^{3}$ is the mean stress scale score.

\section{Discussion}

Unfortunately, we are all currently going through a crisis. The global outbreak of COVID-19 has impacted every-day life in a remarkable way. The daily number of victims continues to rise and many of us remain quarantined at home. COVID-19 does not solely affect individuals who test positive for the virus; the pandemic effect is extremely massive and affects every person, worldwide, as most people are experiencing increased levels of anxiety, stress, and depression, reduced exercise and physical activity, and reduced social interactions due to the compelled lockdown.

The findings of this study revealed normal anxiety levels, and mild depression and stress scores, and mild total DASS scores, with significant differences among the three studied countries, with Egyptian participants demonstrating higher mean scores for depression, anxiety, stress, and total DASS. This study was performed during a period when COVID-19 reporting in Egypt was minimal, with a small number of cases reported daily, and the population may not have had sufficient information regarding this recently developed pandemic. People appear to be more inclined to experience anxiety, depression, and worry when dealing with unknown issues, challenges, or diseases. When humans become more worried, they become more anxious and, thus, more careful. Anxiety reflects worry regarding anticipated danger, and panic is the dissemination of anxiousness among a group. In this context, the anxiety of individuals continuously disseminates, through the speedy transmission of information, advancing into group anxiety and panic. As the reported number of confirmed cases and death associated with COVID-19 increases, the public's psychological status is likely to worsen. However, a mild to moderate level of anxiety is likely to improve people's attention to disease prevention, decreasing the incidence of disease.

These results were congruent with those reported by Leung et al., [34] in a study from Hong Kong, who stated that a positive level of anxiety could encourage the population to take extra preventive measures, decreasing the velocity of SARS transmission. Similarly, a recent study performed in India by Varshney et al. [35] concluded that most of the respondents (66.8\%) experienced minimal psychological distress in response to the COVID-19 outbreak, whereas a small proportion (15\%) experienced mild psychological impacts. In contrast, the findings of this study differed 
from the findings of a study performed in China, by Wang et al., [36] which stated that $53.8 \%$ of respondents suffered from psychological manifestations due to the outbreak, ranging from moderate to severe, in a sample of 1210 respondents.

Several studies have identified relationships between the prevalence of infectious disease outbreaks and a variety of psychological and behavioral consequences. Among the most common negative psychological complications include the increased incidence of depression and psychological misery [37], worry [38], and anxiousness about becoming infected [39]. Furthermore, Kelvin and Rubino [40] in their study, which was performed in China, mentioned that intellectual health problems can manifest, worsen, or trigger psychological and emotional distress in selfisolated and quarantined individuals.

The results of the current study demonstrated that the DASS scores were significantly higher among participants younger than 24 years compared with those older than 45 years. In addition, DASS scores were higher for women, which may be because women are more prone to anxiety than men, due to increased psychological stress regarding both herself and her children, and women tend to be responsible for the social productivity of their families, which may result in the endurance of increased psychological tension. Young individuals were more likely to suffer from symptoms of anxiety, depression, and stress when confronted with epidemics, possibly because young people obtaining massive amounts of information from social and mass media, which could easily trigger and increase psychological distress.

A similar result was reported by Qiu et al., [13] in their survey study of more than 50,000 Chinese respondents, in which nearly $35 \%$ of contributors reported trauma-related distress symptoms, with women and younger adults displaying notably higher levels of psychological distress. Similarly, Cheng et al. [41] and Liu et al. [42] reported higher stress scores among the young adult group (18-30 years), which was consistently associated with a higher risk of different mental health outcomes.

Some evidence has suggested that fluctuations in ovarian hormone levels may be responsible for altered sensitivity to emotional stimuli during specific phases of the menstrual cycle, during which intrusive flashbacks are enhanced and form a foundation that makes women particularly vulnerable to psychological disorders [43].

The results of this study showed that DASS mean scores were higher among participants with family sizes of 4-6 members, and small family sizes were associated with increased risk for the development of depression, stress, and anxiety. This association may represent the increased concern and worry that younger adult with children experiences regarding the potential effects of the COVID-19 outbreak on the health of their children. However, this finding was contradicted by Naushad et al., [44] who concluded that no link exists between having children and psychological consequences, in their review of previous studies.

The WHO speculates that new measures such as self-isolation and quarantine that affect the daily activities, routines, and livelihoods of humans that may result in increased feelings of loneliness, anxiety, and depression, insomnia, dangerous alcohol and drug use, and in some rare cases, deliberate self-harm or suicidal behavior [45].

The results of the present study confirmed a significant association between high DASS mean scores and widowed participants compared with the DASS scores of both married and single participants, which may be due to household financial losses, the lack of psychological support against fear and anxiety emotions or depression symptoms, and a lack of opportunities to communicate with other individuals, which can result in psychological problems, such as despair and anxiety, further highlighting the significance of household support during the course of this emergency. Participants with basic school educations had significantly higher mean DASS scores compared with participants with all other education levels. An individual with a higher educational level may better able to understand the problems associated with posttraumatic stress disorder (PTSD) and psychological distress, allowing these individuals to take positive measures to avoid the development of psychological symptoms, increasing the individual's confidence in mental health recovery. A similar result was reported by Liang et al., [46] who concluded, in their study in China, that younger participants with lower education levels and divorced/widowed individuals were more likely to exhibit PTSD symptoms and psychological distress.

The current study revealed that mean DASS scores were higher among individuals who reported unemployment and insufficient income, which may due to the precautionary measures associated with COVID19 that has limited working conditions and significantly impacted families' financial situations. This result was consistent with the result of a previous study by Brooks et al., [6] who analyzed the role played by financial income and how income changes following labor measures taken for the duration of an epidemic. Thus, decreased or low financial earnings were persistently associated with elevated risks of psychological impacts. Gómez-Salgado et al. [47] reported that general indicators of mental health in Spain suggested that low levels or the lack of financial income and the lack of employment were linked with diminished psychological wellness. Brown and Arnholz [48] reported that, in the United States, businesses and people were required to follow social distancing protocols, maintaining at least 6 feet away from other people, limiting most face-toface interactions, and the unemployment rate due to 
the COVID-19 outbreak could increase the despair and depression levels.

The results of this study demonstrated that DASS mean scores during the course of home quarantine were significantly higher among participants who reported a low appetite, being underweight, and disturbances in sleep, sexuality, and social communication due to quarantine, and among those who were not able to concentrate on positive thoughts during difficulties and did not care about meeting and communicating with family on a daily basis. As the outbreak progressed, with the increasing implementation of restrictive measures, the number of people who became isolated at home, due to lockdown and obligatory domestic quarantine, increased significantly, worldwide, resulting in unexpected, sudden, and radical changes in the habits and lifestyles of the population, including the drastic deterioration of any form of socialization. Physical distancing and selfisolation strongly impacted citizens' lives, affecting food consumption habits and day-to-day behaviors, which subsequently resulted in a variety of negative emotional outcomes.

This result agreed with the results of Lippi et al., [49] in their study showing that the dramatic reduction in physical exercise due to obligatory homestay was one of the most obvious consequences of the general lockdown, not only among those active and energetic individuals who habitually participate in leisure sports but for those who commute to work by walking or cycling and those whose work involves physical activity. Furthermore, Harris and Bargh [50] mentioned that extended shelter-in-place ordinances and sedentary lifestyles will predispose people to weight gain, an issue magnified by the unhealthy dietary habits that very often accompany home setting and extended TV viewing. This result was supported by recent evidence that demonstrated that the sedentary behaviors of younger individuals may also be an essential cause of despair and nervousness [51].

These outcomes were similar to those reported by Huang and Zhao [52], who performed a webbased study that revealed a high risk of generalized anxiety disorder and interrupted sleeping patterns among the Chinese public during the COVID-19 outbreak. In addition, almost one-fifth of participants reported depressive symptoms and sleep problems, indicating that the uncertainty of the epidemic outbreak development may have increased the psychological stress experienced by the public. In contrast, Gleeson et al. [53] revealed that the state of self-isolation, lockdown, and social distancing is essential measures necessary to reduce the curve of the disease, despite the severe consequences that these measures may have on an individual's life. The act of being restricted to one's home has substantial effects on one's health, including modifications to eating and food consumption patterns, sleeping habits, and physical activity. Home quarantine, therefore, promotes sedentary behaviors, which affects both psychological and physical wellness and, subsequently, may lead to greater obesity risk. Fear and anxiety may also induce adjustments in dietary habits, leading to unhealthy dietary patterns, less desire to consume food or less enjoyment during eating [54].

The results of the current study revealed that participants from Egypt displayed a moderate level of self-efficacy during home quarantine, whereas those from Saudi Arabia and Jordan displayed high selfefficacy. Self-efficacy is a reflection of an individual's perception of their capability to engage in protective actions, such as the ability to implement proposed hygienic and protection measures, to stay at home with their household, to reduce leisure and recreational activities, and to protect themselves and prevent illness. High self-efficacy is also an indication people have begun to pay attention to their health and were more likely to seek and find social assistance from their households, as an alternative to meeting with friends, which suggested that the interests and concerns of individuals have been influenced by restricted transport and travel policies and the self-isolation rules established by the health authorities and central governments.

These results were congruent with a previous study, reported by Schwarzer and Hallum [55], who found that self-efficacy distinguishes how people think, feel, and act. According to social cognition theory, human motivation and actions are regulated by forethought. This theory implies that self-efficacy is an independent predictor of behavior, affecting intentions, goals, and outcome expectations, which, in turn, are additional predictors of behavior. Similarly, Barofsky et al., [56] who studied the consequences of fear, the perception of threats, and worry regarding health behaviors, found that higher threat perception will only predict precautionary behaviors when human beings realize and believe that high-quality defensive responses are readily available (known as adequate response efficacy) and when they believe in their own abilities and competencies to engage in such defensive and protective activities (sufficient self-efficacy).

The perceived efficacy of behavior has been assessed and the degree to which respondents felt that their behaviors would effectively protect and guard them against the COVID was evaluated in the current study. In a UK study, an association was identified between perceived efficacy and the performance of preventive behaviors (hand hygiene, adopting flu friend plans, sterilizing, and cleaning surfaces) that protected against swine flu, as reflected by the reports of that robust study [57]. In a Saudi Arabian study, evidence suggested that performing some interventions, such as continuing education, awareness-raising, continuous monitoring processes, and use of reminders and warning signs, can increase the commitment of individuals and health professionals to continued infection control and 
prevention measures, including hand hygiene and the use of alcohol hand rubs [58], which will likely have a positive impact on increasing self-confidence and selfefficacy for dealing with such infectious diseases.

In agreement with other recent studies, Chen et al., [59] stated that few established, medically confirmed measures have been presented that people can implement for the duration of a pandemic to mitigate their risks of contracting the disease. These measures include, but are not limited to, washing hands, minimizing social contacts, the use of protective masks, wearing protective gloves, and cleaning and disinfecting surfaces. These measures have been communicated to people internationally, through news, social media, posters, lectures, and other formal reports, starting in early 2020, when the COVID19 disease emerged as a worldwide issue. In addition to individual-level health protection measures, governments issued some instructions and orders to prevent large gatherings, in addition to placing many cities and areas with pandemic outbreaks in quarantine [60].

The present study identified a highly significant association between increased self-efficacy level and postgraduate education, employment, and appropriate housing conditions. During the current coronavirus pandemic, most educated individuals, experts, and professionals were aware of this infection, the use of accessible preventive measures, the necessity for social distancing, and the need to comply with authorities' initiatives to restrict the dissemination of infection. This result was supported by Bish and Michie [61], in their study examining the demographic and attitudinal determinants of protective behaviors during pandemics, in which they suggested that highly educated and older individuals were more likely to undertake, implement, and abide by disease prevention and avoidant behaviors.

An Australian cross-sectional study that addressed the public intention to comply with quarantine restrictions in the event of pandemic influenza found that distinctly educated citizens and residents reported greater intentions to comply with influenza disease prevention measures [62]. Australia individuals who were employed but unable to work from home were much less likely to report their intent to comply with quarantine restrictions [63]. Another study, performed in Hong Kong, revealed that married individuals reported that they were more likely to comply with quarantine regulations and policies during the catastrophe of an avian influenza outbreak [64].

The results of the current study illustrated a highly significant relationship between increased selfefficacy and the lack of regular exercise, the maintenance of social communications, the ability to concentrate on positive thoughts, the ability to empty one's brain of thoughts at bedtime, and prioritizing meeting and communicating with family on a daily basis. Individuals who participate in regular exercise are likely to suffer during this curfew period, due to restrictions that prevent them from practicing their regular daily exercise; however, individuals who experienced no interruptions in their social communications due to COVID-19 reported better self-efficacy than others, which reflected the importance of social communications for providing psychological support and improving self-efficacy. People who usually concentrate on positive thoughts, who are able to ignore bad thoughts at bedtime, and who regularly discuss their issues with family members are likely to present an improved psychological status, less stress, and higher self-efficacy level than individuals who are unable to perform these acts. In addition, the COVID 19 pandemic may result in negative emotional impacts for the population, increasing depression, anxiety, and distress within the population, which may make some individuals feel safer when at home, as quarantine provides these individuals the perception that they are capable of managing and coping with the current pandemic situation. This result was consistent with the report by Zhang and Ma [65], who found that after the onset of the pandemic, the majority of participants reported paying more attention to their psychiatric and mental health and spent extra time relaxing, praying, resting, and exercising. These advantageous influences on mental and spiritual wellness may have helped individuals cope with the various negative impacts on psychiatric and mental health that can be attributed to the pandemic outbreak and the precautionary measures taken.

Similarly, Thomasson and Psouni [66] reported that a sense of high self-efficacy can be understood as the experience of believing in one's capability to cope with the issues and challenges that arise, which is likely to encourage the active participation and engagement in attempts to manage various problems; therefore, selfefficacy increases the likelihood of engaging in problemfocused coping, rather than becoming dysfunctional.

Individuals who reported a low appetite and sleep and sexual disturbances were also associated with lower self-efficacy levels, which was reflected in the results of the current study. Significantly reduced GSES scores can be attributed to a disturbed psychological and physical status, as those participants reported poor appetite, interrupted sleep, and sexual disturbances associated with the COVID-19 quarantine.

The current study showed a strong negative correlation between DASS and GSES scores, indicating that when the DASS increased, self-efficacy decreased. The mean scores of the depression, anxiety, and stress subscales were identified as independent predictors of GSES scores. A similar result was reported by a study, conducted among a large sample of normal adolescents from the Netherlands, which found that low levels of selfefficacy were highly associated with and accompanied by high levels of trait anxiety, neuroticism, despair, and depressive symptoms in this cohort [67].

Singh et al., [68] recruited 160 elderly Indian respondents and discovered that perceived selfefficacy emerged as an essential predictor of psychiatric and mental health among elderly participants of both genders. The older participants who perceived 
themselves to be self-efficacious and to have control over their surroundings reported better mental health and psychological stability. In another study of adolescents, the self-perception that one has the poor ability to cope with unusual situations and dramatic changes and to lose control during unusual social situations was associated with higher levels of social anxiousness and the increased feeling that anxiety limits one's abilities and has a handicapping effect [69].

\section{Conclusion}

The COVID-19 quarantine has been associated with mild levels of depression stress and normal anxiety levels, with moderate to high self-efficacy levels. Participants from Egypt suffered from greater levels of stress, anxiety, and depression and lower self-efficacy compared with those among individuals from Jordan and Saudi Arabia.

Gender, housing conditions, employment, age, appetite, weight, education, sleeping patterns, sexuality, social communications, work schedules, the ability to concentrate on positive thoughts, the ability to empty thoughts at bedtime, and conversing with family members were associated with depression, anxiety, and stress. Females, poor housing conditions, unemployment, young age, low appetite, being underweight, having a basic school educated, suffering from sleep and sexuality disturbances, having poor social communications, changes in work schedules, the inability to concentrate on positive thoughts, the inability to empty thoughts at bedtime, and not caring about meeting and communicating with family on a daily basis during the COVID-19 quarantine were significantly associated with increased DASS scores.

Gender, housing conditions, loneliness, employment, monthly income, age, marital status, education, exercise, sexuality, social communication, concentrating on positive thoughts, emptying thoughts at bedtime, and conversing with family members were associated with self-efficacy. Males, appropriate housing conditions, living alone, being employed with a sufficient monthly income, older age, being married, higher educational levels, no regular exercise, no sexual or social communication disturbances, the ability to concentrate on positive thoughts, the ability to empty thoughts at bedtime, and caring about meeting and communicating with household members on a daily basis during the COVID-19 quarantine were significantly associated with higher GSES scores.

A significant positive correlation was identified among depression, anxiety, and stress subscales, whereas a significant negative correlation was confirmed between psychological distress and selfefficacy in individuals.

\section{Recommendations}

Additional exploration and focus on the emotional status of individuals during the COVID-19 pandemic is recommended, and awareness programs designed to address the psychological effects of quarantine should be implemented, using mass media and other means. Effectively addressing emotional needs during and after COVID-19, as well as preparing for potential future outbreaks, will require an understanding of the nature and extent of the emotional impacts and the factors linked to negative emotional outcomes during disease outbreaks; thus, the evidence regarding the effectiveness of interventions can be rapidly implemented to prevent and overcome emotional problems that may arise.

The effects of the COVID-19-associated quarantine on emotional status in various populations (general population, COVID-19 cases, close contacts of COVID-19 cases, and healthcare workers) should be carefully examined to design effective intervention strategies that are tailored for each population. However, focusing on improving self-efficacy among the public, in terms of protecting themselves and preventing disease contraction, is likely to play a vital role in improving their emotional status and reducing psychological distress during the pandemic outbreak. Finally, a similar study, conducted using random, larger sample size, should be performed that includes more countries in the region and utilizes a longitudinal research design.

\section{Acknowledgment}

We would like to dedicate this research work to those who have suffered from the COVID-19 virus infection and to the deceased. Furthermore, we would like to dedicate the results of this research study to the health-care providers around the world, who are on the front lines, defending societies against this deadly virus.

\section{References}

1. Centers for Disease Control and Prevention. Quarantine and Isolation 2017. Available from: https://www.cdc.gov/quarantine/ index.html. [Last accessed on 2020 Jan 30].

2. Manuell ME, Cukor J. Mother Nature versus human nature: public compliance with evacuation and quarantine. Disasters. 2011;35(2):417-42. https://doi. org/10.1111/j.1467-7717.2010.01219.x

PMid:21073672

3. Gordon M. Functional health patterns: A structure for assessment. In: Nursing Diagnosis: Process and Aplication. $3^{\text {rd }}$ ed. Saint Louis: Mosby; 1994. p. 69-120. 
4. Poletti P, Ajelli M, Merler S. Risk perception and effectiveness of uncoordinated behavioral responses in an emerging epidemic. Math Biosci. 2012;238(2):80-9. https://doi.org/10.1016/j. mbs.2012.04.003

PMid:22579850

5. Hollingsworth TD, Klinkenberg D, Heesterbeek $H$, Anderson RM. Mitigation strategies for pandemic influenza A: balancing conflicting policy objectives. PLoS Comput Biol. 2011;7(2):e1001076. https://doi.org/10.1371/journal. pcbi.1001076

PMid:21347316

6. Brooks SK, Webster RK, Smith LE, Woodland L, Wessely S, Greenberg N, et al. The psychological impact of quarantine and how to reduce it: Rapid review of the evidence. Lancet. 2020;395:912-20. https://doi.org/10.1016/ S0140-6736(20)30460-8

PMid:32112714

7. Public Health England. Coronavirus (COVID-19) what you need to know: Blog Editor; 2020. Available from: https://www. publichealthmatters.blog.gov.uk/2020/01/23/wuhan-novelcoronavirus-what-you-need-to-know. [Last accessed on 2020 Jun 26].

8. Badran M. What is COVID-19 Quarantine? Dr. Badran Answers: Opinion; 2020. Available from: https://www.see. news/covid-19-quarantine-what-is-quarantine. [Last accessed on 2020 May 12].

9. Xiang YT, Yang Y, Li W, Zhang L, Zhang Q, Cheung T, et al. Timely mental health care for the 2019 novel coronavirus outbreak is urgently needed. Lancet Psychiatry. 2020;7(3):2289. https://doi.org/10.1016/S2215-0366(20)30046-8 PMid:32032543

10. Rubin GJ, Potts $\mathrm{H}$, Michie $\mathrm{S}$. The impact of communications about swine flu (influenza A H1N1v) on public responses to the outbreak: Results from 36 national telephone surveys in the UK. Health Technol Assess. 2010;14(34):183-266. https://doi. org/10.3310/hta14340-03

PMid:20630124

11. Van Bortel T, Basnayake A, Wurie F, Jambai M, Koroma AS, Muana AT, et al. Psychosocial effects of an Ebola outbreak at individual, community and international levels. Bull World Health Organ. 2016;94(3):210-4. https://doi.org/10.2471/ BLT.15.158543

PMid:26966332

12. Bao Y, Sun Y, Meng S, Shi J, Lu L. 2019-nCoV epidemic: Address mental health care to empower society. Lancet. 2020;395(10224):e37-8. https://doi.org/10.1016/ S0140-6736(20)30309-3

PMid:32043982

13. Qiu J, Shen B, Zhao M, Wang Z, Xie B, Xu Y. A nationwide survey of psychological distress among Chinese people in the COVID-19 epidemic: Implications and policy recommendations. Gen Psychiatr. 2020;33(2):e100213. https://doi.org/10.1136/ gpsych-2020-100213 PMid:32215365

14. Bandura A. Efficacy: The Exercise of Control. NewYork: Freeman; 1997. p. 14-139.

15. Bals M, Turi AL, Skre I, Kvernmo S. The relationship between internalizing and externalizing symptoms and cultural resilience factors in Indigenous Sami youth from Arctic Norway. Int J Circumpolar Health. 2011;70(1):37-45. https://doi.org/10.3402/ ijch.v70i1.17790 PMid:21329576

16. Roddenberry A, Renk K. Locus of control and self-efficacy: Potential mediators of stress, illness, and utilization of health services in college students. Child Psychiatry Hum Dev. 2010;41(4):353-70. https://doi.org/10.1007/s10578-010-0173-6

\section{PMid:20204497}

17. Takaki J, Nishi T, Shimoyama $H$, Inada $T$, Matsuyama $N$, Kumano $\mathrm{H}$, et al. Interactions among a stressor, self-efficacy, coping with stress, depression, and anxiety in maintenance hemodialysis patients. Behav Med. 2003;29(3):107-12. https:// doi.org/10.1080/08964280309596063 PMid:15206829

18. Wu A, Tang CS, Kwok T. Self-efficacy, health locus of control, and psychological distress in elderly Chinese women with chronic illnesses. Aging Ment Health. 2004;8(1):21-8. https:// doi.org/10.1080/13607860310001613293

PMid:14690864

19. Jerusalem M, Hessling JK. Mental health promotion in schools by strengthening self-efficacy. Health Educ. 2009;109(4):32941. https://doi.org/10.1108/09654280910970901

20. Terry DJ. Determinants of coping: The role of stable and situational factors. J Pers Soc Psychol. 1994;66(5):895. https:// doi.org/10.1037/0022-3514.66.5.895

PMid:8014834

21. Wikipedia. Saudi Arabia, from Wikipedia, the Free Encyclopedia; 2020. Available from: https://www.en.wikipedia.org/wiki/Saudi Arabia\#cite_note-16. [Last accessed on 2020 Jun 28].

22. Wikipedia. Jordan, from Wikipedia, the Free Encyclopedia 2020. Available from: https://www.en.wikipedia.org/wiki/Jordan. [Last accessed on 2020 Jun 28].

23. Statistics E. Central Agency for Public Mobilization and Statistics; 2020. Available from: http://www.capmas.gov.eg. [Last accessed on 2020 Jun 26].

24. Lovibond PF, Lovibond $\mathrm{SH}$. The structure of negative emotional states: Comparison of the depression anxiety stress scales (DASS) with the beck depression and anxiety inventories. Behav Res Ther. 1995;33(3):335-43. https://doi. org/10.1016/0005-7967(94)00075-u PMid:7726811

25. Le $T A$, Le $M Q$, Dang $A D$, Dang AK, Nguyen $C T$, Pham $H Q$, et al. Multi-level predictors of psychological problems among methadone maintenance treatment patients in difference types of settings in Vietnam. Subst Abuse Treat Prev Policy. 2019;14(1):39. https://doi.org/10.1186/s13011-019-0223-4 PMid:31533764

26. Norton PJ. Depression anxiety and stress scales (DASS-21): Psychometric analysis across four racial groups. Anxiety Stress Coping. 2007;20(3):253-65. https://doi. org/10.1080/10615800701309279 PMid:17999228

27. Henry JD, Crawford JR. The 21-item version of the depression anxiety stress scales (DASS-21): Normative data and psychometric evaluation in a large non-clinical sample. Br J Clin Psychol. 2005;44(22):227-39. https://doi. org/10.1348/014466505X29657

\section{PMid: 16004657}

28. Lovibond PF, Lovibond SH. DASS Frequently Asked Questions, UNSW Psychology 2018. Available from: http://www2.psy. unsw.edu.au/dass//DASSFAQ.htm\# 3. How do_Iget permission_to_use. [Last accessed on 2020 Mar 19].

29. Moussa MT, Lovibond P, Laube R, Megahead HA. Psychometric properties of an arabic version of the depression anxiety stress scales (DASS). Res Soc Work Pract. 2017;27(3):375-86. https:// doi.org/10.1177/1049731516662916

30. Schwarzer R, Jerusalem M. Generalized self-efficacy scale. In: Weinman J, Wright S, Johnston M. Measures in Health Psychology: A User's Portfolio. Causal and Control Beliefs. Windsor, UK: Nfer-Nelson; 1995. p. 35-7. Avaialble from: https:// www.drugsandalcohol.ie/26768/1/General_Self-Efficacy_ Scale\%20(GSE).pdf. [Last accessed on 2020 Feb 20]. 
31. Sbicigo JB, Teixeira MA, Dias AC, Dell'Aglio DD. Propriedades psicométricas da escala de autoeficácia geral percebida (EAGP). Psico. 2012;43(2):1.

32. Schwarzer R, Jerusalem M. General Self-Efficacy Scale (GSE) in 32 Languages; 2012. Available from: http://www. userpage.fu-berlin.de/ health/selfscal.htm. [Last accessed on 2019 Dec 12].

33. General Assembly of the World Medical Association. World medical association declaration of Helsinki: Ethical principles for medical research involving human subjects. J Am Coll Dent. 2014;81(3):14-8. PMid:25951678

34. Leung G, Lam T, Ho L, Ho S, Chan B, Wong I, et al. The impact of community psychological responses on outbreak control for severe acute respiratory syndrome in Hong Kong. J Epidemiol Community Health. 2003;57(11):857-63. https://doi.org/10.1136/ jech.57.11.857

PMid: 14600110

35. Varshney M, Parel JT, Raizada N, Sarin SK. Initial psychological impact of COVID-19 and its correlates in Indian Community: An online (FEEL-COVID) survey. PLoS One. 2020;15(5):e0233874. https://doi.org/10.1371/journal.pone.0233874 PMid:32470088

36. Wang C, Pan R, Wan X, Tan Y, Xu L, Ho CS, et al. Immediate psychological responses and associated factors during the initial stage of the 2019 coronavirus disease (COVID-19) epidemic among the general population in China. Int J Environ Res Public Health. 2020;17(5):1729. https://doi.org/10.3390/ ijerph17051729 PMid:32155789

37. Shultz JM, Cooper JL, Baingana F, Oquendo MA, Espinel Z, Althouse BM, et al. The role of fear-related behaviors in the 2013-2016 West Africa Ebola virus disease outbreak. Curr Psychiatry Rep. 2016;18(104):1-14. https://doi.org/10.1007/ s11920-016-0741-y PMid:27739026

38. Thompson RR, Garfin DR, Holman EA, Silver RC. Distress, worry, and functioning following a global health crisis: A national study of Americans' responses to Ebola. Clin Psychol Sci. 2017;5(3):513-21. https://doi.org/10.1177/2167702617692030

39. Jehn M, Kim Y, Bradley B, Lant T. Community knowledge, risk perception, and preparedness for the 2009 influenza $A / \mathrm{H} 1 \mathrm{~N} 1$ pandemic. J Public Health Manag Pract. 2011;17(5):431-8. https://doi.org/10.1097/PHH.0b013e3182113921 PMid:21788781

40. Kelvin DJ, Rubino S. Fear of the novel coronavirus. J Infect Dev Ctries. 2020;14(1):1-2. https://doi.org/10.3855/jidc.12496 PMid:32088678

41. Cheng $\mathrm{C}$, Jun $\mathrm{H}$, Liang B. Psychological health diathesis assessment system: A nationwide survey of resilient trait scale for Chinese adults. Stud Psychol Behav. 2014;12(6):735-42.

42. Liu N, Zhang F, Wei $C$, Jia $Y$, Shang $Z$, Sun $L$, et al. Prevalence and predictors of PTSS during COVID-19 outbreak in China hardest-hit areas: Gender differences matter. Psychiatry Res. 2020;287:112921. https://doi.org/10.1016/j. psychres.2020.112921 PMid:32240896

43. Soni M, Curran VH, Kamboj SK. Identification of a narrow postovulatory window of vulnerability to distressing involuntary memories in healthy women. Neurobiol Learn Mem. 2013;104:32-8. https://doi.org/10.1016/j.nIm.2013.04.003 PMid:23611942

44. Naushad VA, Bierens JJ, Nishan KP, Firjeeth CP, Mohammad $\mathrm{OH}$, Maliyakkal AM, et al. A systematic review of the impact of disaster on the mental health of medical responders. Prehosp Disaster Med. 2019;34(6):632-43. https:// doi.org/10.1017/S1049023X19004874

PMid:31625487

45. World Health Organization. Mental Health and COVID-19. Geneva: World Health Organization; 2020. Available from: https://www.euro.who.int/en/health-topics/health-emergencies/ coronavirus-covid-19/technical-guidance/mental-health-andcovid-19. [Last accessed on 2020 Jun 20].

46. Liang L, Ren H, Cao R, Hu Y, Qin Z, Li C, et al. The effect of COVID-19 on youth mental health. Psychiatr Q. 2020;91(3):84152. https://doi.org/10.1007/s11126-020-09744-3 PMid:32319041

47. Gómez-Salgado J, Andrés-Villas M, Domínguez-Salas $S$, Díaz-Milanés D, Ruiz-Frutos C. Related health factors of psychological distress during the COVID-19 pandemic in Spain. Int J Environ Res Public Health. 2020;17(11):3947. https://doi. org/10.3390/ijerph17113947 PMid:32498401

48. Brown A, Arnholz J. COVID-19 Jobless Rates will be Comparable to Great Depression: Trump Economic Adviser, Kevin Hassett, the President's Economic Adviser, Appeared on ABC's "This Week"; 2020. Available from: https://www.abcnews. go.com/ThisWeek/covid-19-jobless-rates-comparable-greatdepression-trump/story?id=70348765. [Last accessed on 2020 Apr 26].

49. Lippi G, Henry BM, Bovo C, Sanchis-Gomar F. Health risks and potential remedies during prolonged lockdowns for coronavirus disease 2019 (COVID-19). Diagnosis (Berl). 2020;7(2):85-90. https://doi.org/10.1515/dx-2020-0041

PMid:32267243

50. Harris JL, Bargh JA. Television viewing and unhealthy diet: Implications for children and media interventions. Health Commun. 2009;24(7):660-73. https://doi. org/10.1080/10410230903242267 PMid:20183373

51. Bélair MA, Kohen DE, Kingsbury M, Colman I. Relationship between leisure time physical activity, sedentary behaviour and symptoms of depression and anxiety: Evidence from a population-based sample of Canadian adolescents. BMJ Open. 2018;8(10):e021119. https://doi.org/10.1136/ bmjopen-2017-021119 PMid:30337306

52. Huang Y, Zhao N. Generalized anxiety disorder, depressive symptoms and sleep quality during COVID-19 outbreak in China: A web-based cross-sectional survey. Psychiatry Res. 2020;288:112954. https://doi.org/10.1016/j. psychres.2020.112954 PMid:32325383

53. Gleeson M, Nieman DC, Pedersen BK. Exercise, nutrition and immune function. J Sports Sci. 2004;22(1):115-25. https://doi. org/10.1080/0264041031000140590

PMid: 14971437

54. Macht M. How emotions affect eating: A five-way model. Appetite. 2008;50(1):1-11. https://doi.org/10.1016/j.appet.2007.07.002 PMid: 17707947

55. Schwarzer R, Hallum S. Perceived teacher self-efficacy as a predictor of job stress and burnout: Mediation analyses. Appl Psychol. 2008;57(s1):152-71. https://doi. org/10.1111/j.1464-0597.2008.00359.x

56. Barofsky I, Erickson P, Eberhardt M. Comparison of a single global item and an index of a multi-item health status measure among persons with and without diabetes in the US. Qual Life Res. 2004;13(10):1671-81. https://doi.org/10.1007/s11136-004-0258-4 PMid: 15651538

57. Rubin GJ, Amlôt R, Page L, Wessely S. Public perceptions, 
anxiety, and behaviour change in relation to the swine flu outbreak: Cross sectional telephone survey. BMJ. 2009;339:b2651. https://doi.org/10.1136/bmj.b2651

PMid:19574308

58. Shahin MA. Compliance with hand hygiene among health care providers: Effects of a six sigma improvement project. Int $J$ Public Health Clin Sci. 2018;5(3):112-24.

59. Chen H, Guo J, Wang C, Luo F, Yu X, Zhang W, et al. Clinical characteristics and intrauterine vertical transmission potential of COVID-19 infection in nine pregnant women: A retrospective review of medical records. Lancet. 2020;395(10226):809-15. https://doi.org/10.1016/S0140-6736(20)30360-3

PMid:32213320

60. Remuzzi A, Remuzzi G. COVID-19 and Italy: What next? Lancet. 2020;395:1225-8. https://doi.org/10.1016/ S0140-6736(20)30627-9

PMid:32178769

61. Bish A, Michie S. Demographic and attitudinal determinants of protective behaviours during a pandemic: A review. $\mathrm{Br} J$ Health Psychol. 2010;15(4):797-824. https://doi. org/10.1348/135910710X485826

PMid:20109274

62. Barr M, Raphael B, Taylor M, Stevens G, Jorm L, Giffin M, et al. Pandemic influenza in Australia: using telephone surveys to measure perceptions of threat and willingness to comply. BMC Infect Dis. 2008;8(1):117. https://doi. org/10.1186/1471-2334-8-117

PMid:18793441

63. Eastwood K, Durrheim D, Francis JL, d'Espaignet ET, Duncan S, Islam $\mathrm{F}$, et al. Knowledge about pandemic influenza and compliance with containment measures among Australians. Bull World Health Organ. 2009;87:588-94. https://doi.org/10.2471/ BLT.08.060772

PMid: 19705008

64. Lau JT, Kim JH, Tsui HY, Griffiths S. Anticipated and current preventive behaviors in response to an anticipated humanto-human H5N1 epidemic in the Hong Kong Chinese general population. BMC Infect Dis. 2007;7(1):18. https://doi. org/10.1186/1471-2334-7-18

PMid: 17359545

65. Zhang Y, Ma ZF. Impact of the COVID-19 pandemic on mental health and quality of life among local residents in Liaoning Province, China: A cross-sectional study. Int J Environ Res Public Health. 2020;17(7):2381. https://doi.org/10.3390/ ijerph17072381

PMid:32244498

66. Thomasson P, Psouni E. Social anxiety and related social impairment are linked to self-efficacy and dysfunctional coping. Scand J Psychol. 2010;51(2):171-8. https://doi. org/10.1111/j.1467-9450.2009.00731.x PMid:19500297

67. Muris P. Relationships between self-efficacy and symptoms of anxiety disorders and depression in a normal adolescent sample. Pers Individ Dif. 2002;32(2):337-48. https://doi. org/10.1016/S0191-8869(01)00027-7

68. Singh AP, Shukla A, Singh PA. Perceived self efficacy and mental health among elderly. Delhi Psychiatry J. 2010;13(2):314-21.

69. Gaudiano BA, Herbert JD. Self-efficacy for social situations in adolescents with generalized social anxiety disorder. Behav Cogn Psychother. 2007;35(2):209-23. https://doi.org/10.1017/ S1352465806003377 